OPEN ACCESS

Edited by:

Soo-Kyoung Choi,

Yonsei University College of Medicine,

South Korea

Reviewed by:

Jane A. Leopold,

Brigham and Women's Hospital and Harvard Medical School,

United States

Yi Song,

Peking University, China

Zhiyong Zou,

Peking University, China

*Correspondence:

Peige Song

peigesong@zju.edu.cn

tThese authors have contributed equally to this work

Specialty section:

This article was submitted to

Hypertension,

a section of the journal

Frontiers in Cardiovascular Medicine

Received: 10 November 2020 Accepted: 08 April 2021

Published: 11 May 2021

Citation:

Ye X, Yi Q, Shao J, Zhang Y, Zha M,

Yang $Q$, Xia $W, Y e Z$ and Song $P$

(2021) Trends in Prevalence of

Hypertension and Hypertension Phenotypes Among Chinese Children and Adolescents Over Two Decades

(1991-2015).

Front. Cardiovasc. Med. 8:627741. doi: 10.3389/fcrm.2021.627741

\section{Trends in Prevalence of Hypertension and Hypertension Phenotypes Among Chinese Children and Adolescents Over Two Decades (1991-2015)}

\author{
Xinxin Ye ${ }^{1+}$, Qian $\mathrm{Yi}^{1+}$, Jing Shao ${ }^{2}$, Yan Zhang ${ }^{3}$, Mingming Zha ${ }^{4}$, Qingwen Yang ${ }^{4}$, Wei Xia ${ }^{5}$, \\ Zhihong $\mathrm{Ye}^{2}$ and Peige Song ${ }^{1,6 *}$
}

\begin{abstract}
${ }^{1}$ School of Public Health, Zhejiang University School of Medicine, Zhejiang University, Hangzhou, China, ${ }^{2}$ School of Nursing, Zhejiang University School of Medicine, Zhejiang University, Hangzhou, China, ${ }^{3}$ Faculty of Life Science and Medicine, Kings College London, London, United Kingdom, ${ }^{4}$ Medical School Southeast University, Nanjing, China, ${ }^{5}$ School of Nursing, Sun Yat-Sen University, Guangdong, China, ${ }^{6}$ Women's Hospital, Zhejiang University School of Medicine, Zhejiang University, Hangzhou, China
\end{abstract}

Background: Hypertension is a leading cause of cardiovascular-related morbidity and mortality. Elevated blood pressure (BP) in children is related to long-term adverse health effects. Until recently, few studies have reported the secular trend and associated factors of hypertension phenotypes in Chinese children and adolescents.

Methods: From the China Health and Nutrition Survey (CHNS) 1991-2015, a total of 15,143 records of children aged 7-17 years old were included. Following definitions of hypertension from the Chinese Child Blood Pressure References Collaborative Group, we estimated the prevalence of prehypertension, hypertension, stage 1 hypertension, stage 2 hypertension and its phenotypes (ISH, isolated systolic hypertension; $\mathrm{IDH}$, isolated diastolic hypertension; SDH, combined systolic and diastolic hypertension). General estimation equation was used to analyze the trends in the prevalence of hypertension and hypertension phenotypes, and a multivariable logistic regression was constructed to explore the associated factors.

Results: During 1991-2015, increasing trends were revealed in BP and hypertension prevalence $(P<0.001)$ in Chinese children and adolescents. For ISH, IDH and SDH, the age-standardized prevalence increased dramatically from 0.9 to $2.2 \%$, from 6.2 to $14.1 \%$, and from 1.4 to $2.9 \%$, respectively (all $P<0.001$ ). Adolescents aged 13-17 years $(\mathrm{OR}=1.76,95 \% \mathrm{Cl}: 1.56-1.97, P<0.001)$, general obesity $(\mathrm{OR}=2.69,95 \% \mathrm{Cl}$ : 2.10-3.44, $P<0.001)$ and central obesity $(\mathrm{OR}=1.49,95 \% \mathrm{Cl}: 1.21-1.83, P<0.001)$ were positively associated with hypertension, whereas the South region $(\mathrm{OR}=0.65$, 95\% Cl: 0.58-0.73, $P<0.001)$ was a negative factor. Furthermore, body mass index (BMI) and general obesity were linked to the presence of ISH, IDH and SDH. Age, waist circumference (WC) and central obesity were additionally associated with ISH, and sex, age, urban/rural setting, North/South region, WC and central obesity were additionally associated with IDH. 


\begin{abstract}
Conclusion: BP levels and prevalence of hypertension and phenotypes increased dramatically in Chinese children and adolescents from 1991 to 2015. Regional discrepancy, demographic features, BMI, WC and overweight/obesity status were associated factors of hypertension among youths.
\end{abstract}

Keywords: hypertension, hypertension phenotypes, China, children, adolescents, trends

\section{INTRODUCTION}

Hypertension, or elevated blood pressure (BP), is a highly prevalent chronic disease globally. Hypertension has been recognized as a primary modifiable contributor to cardiovascular and cerebrovascular diseases (1). In China, hypertension affects more than 270 million people, emerging as a major public health challenge (2-4). It was estimated that the direct costs caused by hypertension reached 210.3 billion Yuan in 2013, accounting for $6.61 \%$ of the total Chinese health-care expenditure (5). Hypertension is not a disorder that confines to adults, globally, millions of children were suffering from hypertension (6). Pathophysiologic and epidemiologic evidence has shown an association between childhood hypertension and long-term adverse health effects (7).

Unlike adulthood hypertension, whose definition has been upgraded to a universally diagnostic standard, the measurement of childhood hypertension is comparatively complicated (8). The cutoffs of childhood hypertension have been suggested as a systolic blood pressure (SBP) or a diastolic blood pressure (DBP) equal to or higher than 95th percentile by age, sex, and height (9). According to the fourth report from the National High Blood Pressure Education Program (NHBPEP) Working Group in the United States, BP in children and adolescents was suggested to be measured on at least three separate occasions at an interval of 2 weeks due to the apparent fluctuations (10). In 2017, the American Academy of Pediatrics (AAP) guidelines recommended the use of ambulatory $\mathrm{BP}$ monitoring in the diagnosis and management of hypertension in children (11).

China is the largest developing country across the world, where the emergence of hypertension has brought a heavy burden to the whole country $(8,10,12,13)$. A cohort study in China revealed that the hypertension prevalence was $17.00 \%$ in boys and $14.13 \%$ in girls aged $7-17$ years (14), and it ranged from 5.2 to $7.8 \%$ in Italy among school-aged children $(15,16)$. Several previous studies have focused on the prevalence of hypertension, whereas studies on trends in different hypertension phenotypes were limited in China. Early identification of different hypertension phenotypes in youth is of significant importance in preventing cardiovascular events in adulthood (17). Compared with previous studies, we extended the study period to 1991-2015. We additionally assessed the prevalence of hypertension severity (stage one hypertension and stage two hypertension) and phenotypes (ISH, isolated systolic hypertension; IDH, isolated diastolic hypertension; SDH, systolic and diastolic hypertension) by age and sex, and explored the effects of demographic, geographic, anthropometric factors and obesity on childhood hypertension in China.
We herein hypothesized that the prevalence of hypertension and hypertension phenotypes in children increased gradually with years; the secular trends in childhood hypertension prevalence differed by age, sex, locations, and region; demographic, anthropometric, geographic factors, and general/central obesity were independently associated with increased BP and hypertension phenotypes in Chinese children and adolescents.

\section{MATERIALS AND METHODS}

\section{Study Design and Study Population}

Details about the study design of CHNS are available elsewhere $(18,19)$. In brief, CHNS is a longitudinal health and nutrition survey in China, beginning in 1989 and having been continuously conducted every 2 or 4 years. So far, CHNS has been conducted for 10 rounds $(1989,1991,1993,1997,2000,2004,2006,2009$, 2011, and 2015) and covered a set of large provinces across China (the list and locations of investigated provinces are shown in Supplementary Table 1 and Supplementary Figure 1). In each round, a multistage random-cluster sampling method was adopted to ensure a good representative of the general Chinese population. First, all counties and cities in each province were stratified into three groups (low-, middle- and high-income). Then, four counties (one low-, two middle-, and one high-income county) and two cities (usually the provincial capital and a lowerincome city) were randomly selected. Third, one community and three rural villages within each selected county and two communities and two suburban villages within each selected city were randomly chosen. In each community or village, all members of 20 households were randomly selected $(18,19)$. A total of 15,143 records (7,423 subjects) aged 7-17 years old with complete data from 1991 to 2015 were included in this study, and the number of participants who participated twice or more was 4,865 . The sample sizes of the nine rounds were 2,429 in 1991, 2,254 in 1993,2,254 in 1997, 2,217 in 2000, 1,364 in 2004, 1,145 in 2006, 1,012 in 2009, 1,404 in 2011 and 1,064 in 2015 (the excluded and included records are shown in Table 1). CHNS was approved by the Institutional Review Committees of the University of North Carolina at Chapel Hill, the National Institute of Nutrition and Food Safety, the Chinese Center for Disease Control and Prevention, and the China-Japan Friendship Hospital, Ministry of Health. All respondents signed informed consent.

\section{Data Collection}

Data on demographics (age and sex) and geographic location (urban/rural and North/South region) were collected by trained interviewers using a structured questionnaire. BP, weight, height, 
TABLE 1 | Comparison of demographic characteristics between the excluded and included subjects in CHNS 1991-2015.

\begin{tabular}{|c|c|c|c|c|c|c|c|c|c|c|c|c|c|c|c|}
\hline \multirow[t]{2}{*}{ Characteristic } & \multicolumn{3}{|c|}{ 1991-2015 combined } & \multicolumn{3}{|c|}{1991} & \multicolumn{3}{|c|}{1993} & \multicolumn{3}{|c|}{1997} & \multicolumn{3}{|c|}{2000} \\
\hline & $\begin{array}{c}\text { Excluded } \\
3,460\end{array}$ & $\begin{array}{c}\text { Included } \\
15,143\end{array}$ & $\begin{array}{c}p \\
\text { value }\end{array}$ & $\begin{array}{c}\text { Excluded } \\
508\end{array}$ & $\begin{array}{c}\text { Included } \\
2,429\end{array}$ & $\begin{array}{c}P \\
\text { value }\end{array}$ & $\begin{array}{c}\text { Excluded } \\
527\end{array}$ & $\begin{array}{c}\text { Included } \\
2,254\end{array}$ & $\begin{array}{c}p \\
\text { value }\end{array}$ & $\begin{array}{c}\text { Excluded } \\
622\end{array}$ & $\begin{array}{c}\text { Included } \\
2,254\end{array}$ & $\begin{array}{c}p \\
\text { value }\end{array}$ & $\begin{array}{c}\text { Excluded } \\
660\end{array}$ & $\begin{array}{c}\text { Included } \\
2,217\end{array}$ & $\begin{array}{c}P \\
\text { value }\end{array}$ \\
\hline \multicolumn{16}{|l|}{ AGE GROUP } \\
\hline $7-12$ years & $\begin{array}{c}1,526 \\
(44.10 \%)\end{array}$ & $\begin{array}{c}8,783 \\
(58.00 \%)\end{array}$ & $<0.001$ & $229(45.08 \%)$ & $\begin{array}{c}1,335 \\
(54.96 \%)\end{array}$ & $<0.001$ & $246(46.68 \%)$ & $\begin{array}{c}1,285 \\
(57.01 \%)\end{array}$ & $<0.001$ & $271(43.57 \%)$ & $\begin{array}{c}1,358 \\
(60.25 \%)\end{array}$ & $<0.001$ & $207(31.36 \%)$ & $\begin{array}{c}1,207 \\
(54.44 \%)\end{array}$ & $<0.001$ \\
\hline $13-17$ years & $\begin{array}{c}1,934 \\
(55.90 \%)\end{array}$ & $\begin{array}{c}6,360 \\
(42.00 \%)\end{array}$ & & 279 (54.92\%) & $\begin{array}{c}1,094 \\
(45.04 \%)\end{array}$ & & 281 (53.32\%) & 969 (42.99\%) & & 351 (56.43\%) & 896 (39.75\%) & & $453(68.64 \%)$ & $\begin{array}{c}1,010 \\
(45.56 \%)\end{array}$ & \\
\hline \multicolumn{16}{|l|}{ SEX } \\
\hline Male & $\begin{array}{c}1,842 \\
(53.24 \%)\end{array}$ & $\begin{array}{c}7,947 \\
(52.48 \%)\end{array}$ & 0.421 & 277 (54.53\%) & $\begin{array}{c}1,251 \\
(51.50 \%)\end{array}$ & 0.215 & $256(48.58 \%)$ & $\begin{array}{c}1,175 \\
(52.13 \%)\end{array}$ & 0.142 & $333(53.54 \%)$ & $\begin{array}{c}1,192 \\
(52.88 \%)\end{array}$ & 0.773 & $342(51.82 \%)$ & $\begin{array}{c}1,176 \\
(53.04 \%)\end{array}$ & 0.58 \\
\hline Female & $\begin{array}{c}1,618 \\
(46.76 \%)\end{array}$ & $\begin{array}{c}7,196 \\
(47.52 \%)\end{array}$ & & $231(45.47 \%)$ & $\begin{array}{c}1,178 \\
(48.50 \%)\end{array}$ & & $271(51.42 \%)$ & $\begin{array}{c}1,079 \\
(47.87 \%)\end{array}$ & & $289(46.46 \%)$ & $\begin{array}{c}1,062 \\
(47.12 \%)\end{array}$ & & $318(48.18 \%)$ & $\begin{array}{c}1,041 \\
(46.96 \%)\end{array}$ & \\
\hline \multicolumn{16}{|l|}{ LOCATION } \\
\hline Urban & $866(25.03 \%)$ & $\begin{array}{c}4,347 \\
(28.71 \%)\end{array}$ & $<0.001$ & $112(22.05 \%)$ & $620(25.52 \%)$ & 0.099 & $110(20.87 \%)$ & $566(25.11 \%)$ & 0.041 & $142(23.00 \%)$ & 659 (29.24\%) & 0.002 & $161(24.39 \%)$ & $609(27.47 \%)$ & 0.117 \\
\hline Rural & $\begin{array}{c}2,594 \\
(74.97 \%)\end{array}$ & $\begin{array}{c}10,796 \\
(71.29 \%)\end{array}$ & & $396(77.95 \%)$ & $\begin{array}{c}1,809 \\
(74.48 \%)\end{array}$ & & $417(79.13 \%)$ & $\begin{array}{c}1,688 \\
(74.89 \%)\end{array}$ & & $480(77.00 \%)$ & $\begin{array}{c}1,595 \\
(70.76 \%)\end{array}$ & & $499(75.61 \%)$ & $\begin{array}{c}1,608 \\
(72.53 \%)\end{array}$ & \\
\hline \multicolumn{16}{|l|}{ REGION } \\
\hline North & $\begin{array}{c}1,434 \\
(41.45 \%)\end{array}$ & $\begin{array}{c}5,286 \\
(34.91 \%)\end{array}$ & $<0.001$ & $226(44.49 \%)$ & $728(29.97 \%)$ & $<0.001$ & $228(43.26 \%)$ & $698(30.97 \%)$ & $<0.001$ & $221(35.53 \%)$ & 776 (34.43\%) & 0.609 & 266 (40.3\%) & $964(43.48 \%)$ & 0.147 \\
\hline South & $\begin{array}{c}2,026 \\
(58.55 \%)\end{array}$ & $\begin{array}{c}9,857 \\
(65.09 \%)\end{array}$ & & 282 (55.51\%) & $\begin{array}{c}1,701 \\
(70.03 \%)\end{array}$ & & 299 (56.74\%) & $\begin{array}{c}1556 \\
(69.03 \%)\end{array}$ & & 401 (64.47\%) & $\begin{array}{c}1,478 \\
(65.57 \%)\end{array}$ & & 394 (59.70\%) & $\begin{array}{c}1,253 \\
(56.52 \%)\end{array}$ & \\
\hline BMI $\left(\mathrm{kg} / \mathrm{m}^{2}\right)$ & $17.42 \pm 4.63$ & $17.69 \pm 3.25$ & 0.040 & $15.4 \pm 1.86$ & $17.38 \pm 2.78$ & $<0.001$ & $15.66 \pm 1.94$ & $17.28 \pm 2.68$ & $<0.001$ & $15.66 \pm 2.05$ & $17.43 \pm 2.78$ & $<0.001$ & $16.46 \pm 2.41$ & $17.68 \pm 2.85$ & 0.004 \\
\hline WC (cm) & $59.01 \pm 12.85$ & $62.87 \pm 9.9$ & $<0.001$ & NA & NA & & $56.48 \pm 6.8$ & $62.39 \pm 8.5$ & $<0.001$ & $55.98 \pm 6.45$ & $61.39 \pm 8.17$ & $<0.001$ & $58.42 \pm 7.12$ & $62.8 \pm 8.38$ & $<0.001$ \\
\hline \multicolumn{16}{|c|}{ GENERAL OBESITY } \\
\hline Normal & $568(85.80 \%)$ & $\begin{array}{c}13,228 \\
(88.06 \%)\end{array}$ & 0.08 & $83(94.32 \%)$ & $\begin{array}{c}2,222 \\
(92.20 \%)\end{array}$ & 0.47 & $86(93.48 \%)$ & $\begin{array}{c}2,046 \\
(91.96 \%)\end{array}$ & 0.60 & $101(93.52 \%)$ & $\begin{array}{c}2,042 \\
(91.12 \%)\end{array}$ & & 44 (95.65\%) & $\begin{array}{c}1,983 \\
(90.05 \%)\end{array}$ & 0.21 \\
\hline $\begin{array}{l}\text { Overweight/ } \\
\text { Obesity }\end{array}$ & $94(14.20 \%)$ & $\begin{array}{c}1,794 \\
(11.94 \%)\end{array}$ & & $5(5.68 \%)$ & $188(7.80 \%)$ & & $6(6.52 \%)$ & 179 (8.04\%) & & $7(6.48 \%)$ & 199 (8.88\%) & 0.39 & $2(4.35 \%)$ & 219 (9.95\%) & \\
\hline \multicolumn{16}{|c|}{ CENTRAL OBESITY } \\
\hline Normal & $567(91.60 \%)$ & $\begin{array}{c}10,571 \\
(91.68 \%)\end{array}$ & 0.94 & NA & NA & & 66 (95.65\%) & $\begin{array}{c}1,217 \\
(96.36 \%)\end{array}$ & 0.76 & $128(96.24 \%)$ & $\begin{array}{c}2,078 \\
(96.25 \%)\end{array}$ & 1.00 & 72 (94.74\%) & $\begin{array}{c}2,068 \\
(94.65 \%)\end{array}$ & 0.97 \\
\hline $\begin{array}{l}\text { Central } \\
\text { Obesity }\end{array}$ & 52 (8.40\%) & 959 (8.32\%) & & NA & NA & & $3(4.35 \%)$ & 46 (3.64\%) & & 5 (3.76\%) & 81 (3.75\%) & & 4 (5.26\%) & 117 (5.35\%) & \\
\hline
\end{tabular}


TABLE 1 | Continued

\begin{tabular}{|c|c|c|c|c|c|c|c|c|c|c|c|c|c|c|c|}
\hline \multirow[t]{2}{*}{ Characteristis } & \multicolumn{3}{|c|}{2004} & \multicolumn{3}{|c|}{2006} & \multicolumn{3}{|c|}{2009} & \multicolumn{3}{|c|}{2011} & \multicolumn{3}{|c|}{2015} \\
\hline & $\begin{array}{c}\text { Excluded } \\
283\end{array}$ & $\begin{array}{c}\text { Included } \\
1,364\end{array}$ & $\begin{array}{c}p \\
\text { value }\end{array}$ & $\begin{array}{c}\text { Excluded } \\
153\end{array}$ & $\begin{array}{c}\text { Included } \\
1,145\end{array}$ & $\begin{array}{c}P \\
\text { value }\end{array}$ & $\begin{array}{c}\text { Excluded } \\
127\end{array}$ & $\begin{array}{c}\text { Included } \\
1,012\end{array}$ & $\begin{array}{c}P \\
\text { value }\end{array}$ & $\begin{array}{c}\text { Excluded } \\
88\end{array}$ & $\begin{array}{c}\text { Included } \\
1,404\end{array}$ & $\begin{array}{c}p \\
\text { value }\end{array}$ & $\begin{array}{c}\text { Excluded } \\
500\end{array}$ & $\begin{array}{c}\text { Included } \\
1,064\end{array}$ & $\begin{array}{c}P \\
\text { value }\end{array}$ \\
\hline
\end{tabular}

\section{AGE GROUP}

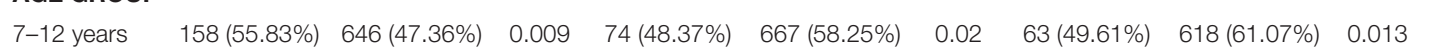

$79(51.63 \%) \quad 478(41.75 \%)$

$64(50.39 \%) \quad 394(38.93 \%)$

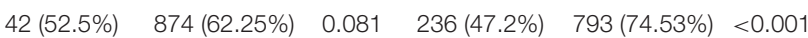

Male $\quad 154(54.42 \%) \quad 720(52.79 \%) \quad 0.617 \quad 85(55.56 \%) \quad 609(53.19 \%) \quad 0.581$

$\begin{array}{ll}85(55.56 \%) & 609(53.19 \%) \\ 68(44.44 \%) & 536(46.81 \%)\end{array}$

$72(56.69 \%) \quad 562(55.53 \%) \quad 0.804$

$38(47.5 \%) \quad 530(37.75 \%)$

$264(52.8 \%) \quad 271(25.47 \%)$

\section{LOCATION}

Urban

$29(45.58 \%) \quad 644(47.21 \%)$

$55(43.31 \%) \quad 450(44.47 \%)$

$39(48.75 \%) \quad 691(49.22 \%)$

$282(56.4 \%) \quad 549(51.60 \%) \quad 0.047$

Rural

$59(20.85 \%) \quad 408(29.91 \%) \quad 0.002$

$224(79.15 \%) \quad 956(70.09 \%)$

$41(26.80 \%) \quad 343(29.96 \%) \quad 0.421$

$35(27.56 \%) \quad 274(27.08 \%) \quad 0.908$

REGION

North

South

$138(48.76 \%) \quad 556(40.76 \%) \quad 0.013$

$12(73.20 \%) 802(70.04 \%)$

$92(72.44 \%) \quad 738(72.92 \%)$

$34(42.50 \%) \quad 530(37.75 \%) \quad 0.395 \quad 172(34.40 \%) \quad 338(31.77 \%)$

$46(57.50 \%) \quad 874(62.25 \%)$

$328(66.00 \%) 726(68.23 \%)$

$\begin{array}{lllllllllll} & 145(51.24 \%) & 808(59.24 \%) & 76(49.67 \%) & 700(61.14 \%) & 87(68.50 \%) & 637(62.94 \%) & 45(56.25 \%) & 962(68.52 \%) & & \end{array}$

$\begin{array}{llllllllllll} & & \end{array}$

GENERAL OBESITY

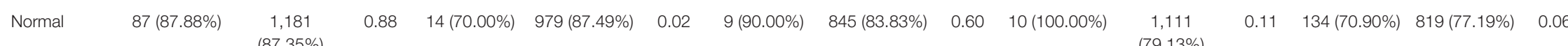

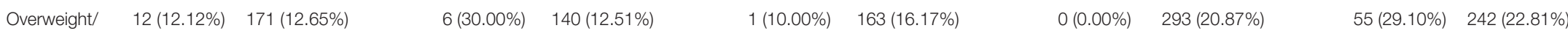

Obesity

CENTRAL OBESITY

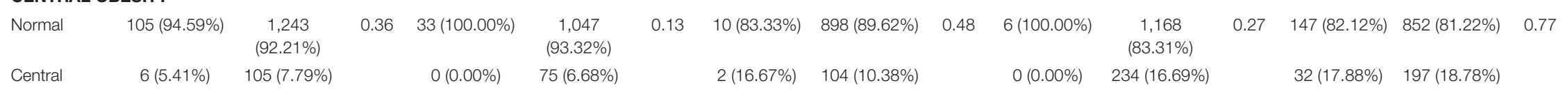

Obesity

Data are presented as means \pm standard deviations or n (\%); BMI, Body Mass Index; WC, Waist Circumference. 
TABLE 2 | Classification of blood pressure and definitions of hypertension and hypertension phenotypes in children and adolescents.

\begin{tabular}{ll}
\hline Category & Definition \\
\hline Normal & SBP with DBP less the 90th percentile (for age, sex and \\
& height) \\
Prehypertension & An SBP and/or DBP between the 90th and 95th \\
& percentile (for age, sex and height) or $\geq 120 / 80 \mathrm{~mm}$ Hg \\
Hypertension & An SBP and/or DBP $\geq 95$ th percentile (for age, sex and \\
& height) on $\geq 3$ separate occasions \\
Stage 1 & An SBP and/or DBP between 95th and 99th percentile \\
& plus 5 mmHg (for age, sex and height) on $\geq 3$ separate \\
& occasions \\
Stage 2 & An SBP and/or DBP above 99th percentile plus 5 mmHg \\
& (for age, sex and height) on $\geq 3$ separate occasions \\
ISH & An SBP $\geq 95$ th percentile (for age, sex, and height) but a \\
& DBP $<95$ th percentile (for age, sex, and height) on $\geq 3$ \\
& separate occasions \\
& A DBP $\geq 95$ th percentile (for age, sex, and height) but an \\
& SBP $<95$ th percentile (for age, sex, and height) on $\geq 3$ \\
separate occasions & An SBP and DBP $\geq 95$ th percentile (for age, sex, and \\
IDH & height) on $\geq 3$ separate occasions \\
SDH &
\end{tabular}

* SBP, systolic blood pressure; DBP, diastolic blood pressure; IDH, isolated diastolic hypertension; ISH, isolated systolic hypertension; SDH, systolic-diastolic hypertension.

waist circumference (WC) and hip circumference (HC) were measured following standardized protocols from the World Health Organization (20). BP was calculated as the mean of three measurements with an interval of 3-5 min by standard mercury sphygmomanometer $(11,21,22)$. Weight was measured without coats to the nearest $0.1 \mathrm{~kg}$ on a calibrated beam scale, and height was measured without shoes to the nearest $0.1 \mathrm{~cm}$ using a portable stadiometer. WC was taken at the end of expiratory period at the midpoint of the line between the lower rib and the upper iliac crest $(23,24)$. HC was measured at the level of maximal gluteal protrusion with a non-elastic tape. Body mass index (BMI) was calculated as weight divided by height squared $\left(\mathrm{kg} / \mathrm{m}^{2}\right)$, waist to height ratio (WHtR) as WC divided by height, and waist to hip ratio (WHR) as WC divided by HC.

\section{Definitions}

\section{Hypertension and Phenotypes}

Conforming to the standard of the Chinese Child Blood Pressure References Collaborative Group, the definitions of childhood hypertension and phenotypes are listed in Table 2 (25).

\section{Demographics, Geographic Locations, General, and Central Obesity}

Following the criteria of the 2017 AAP guidelines, we divided all participants into two age groups of 7-12 and 13-17 years (11). Residence was classified into South China (Shanghai, Jiangsu, Hubei, Hunan, Guangxi, Guizhou, and Chongqing in CHNS) and North China (Beijing, Liaoning, Heilongjiang, Shandong, and Henan in CHNS). Using the Working Group on Obesity in China criteria, a BMI between 85th and 95th percentile of sex and age group was defined as overweight, and $\geq 95$ th percentile as obesity. Central obesity was defined as a $\mathrm{WC}>90$ th percentile in each sex and age group, or a WHtR $\geq 0.5$, or a WHR $\geq 0.9$ in boys or $\geq 0.85$ in girls (26).

\section{Statistical Analysis}

In descriptive analyses, continuous variables were reported as mean and standard deviation (SD) and categorical data were presented as percentage (\%) with $95 \%$ confidence interval (CI). Using the Chi-square test, we compared the age and sex distributions between the excluded and included groups. Given that the same participant might be included in different survey rounds, generalized estimating equation (GEE) was adopted (27, 28). Individual calendar year was selected as a single continuous variable and age were adjusted for in GEE models to examine the trends in the prevalence of childhood hypertension and phenotypes. Subgroup trend analyses from 1991 to 2015 were conducted by strata of age and sex. To estimate the associated factors of childhood hypertension and phenotypes, multivariable logistic regression was carried out. All statistical analyses were performed using Stata statistical software (version 14.0; Stata Corporation, College Station, TX, USA), and a $P<0.05$ was considered as statistically significant in two-sided tests.

\section{RESULTS}

\section{Characteristics of the Participants}

A total of 18,603 records of children and adolescents were available from CHNS 1991 to 2015, of which 15,143 were with BP measurements. The basic characteristics (demographics, anthropometry and geography) are shown in Table 3. The flow chart of this study is shown in Supplementary Figure 2.

\section{Trends in SBP, DBP, and Prevalence of Childhood Hypertension and Phenotypes}

Table 4 shows the changes in age-standardized mean SBP and DBP by strata of age and sex. From 1991 to 2015, SBP and DBP values increased significantly from $96.18 \mathrm{mmHg}$ (95\% CI: 95.65-96.71) to $101.54 \mathrm{mmHg}$ (95\% CI: 100.78-102.30), and from $62.61 \mathrm{mmHg}$ (95\% CI: $62.22-63.01)$ to $66.65 \mathrm{mmHg}(95 \%$ CI: 66.10-67.20), respectively (both $P$-values for age-adjusted trend $<0.001)$. Mean SBP and DBP values increased across age and sex groups during the same time period, with an average annual increase (AAI) ranging from $0.12 \mathrm{mmHg}$ to $0.37 \mathrm{mmHg}$ and an average relative increase (ARI) from 0.19 to $0.46 \%$.

Table 5 presents the trends of age-standardized prevalence of childhood hypertension and phenotypes. For prehypertension, the age-standardized prevalence significantly increased from 7.0\% (95\% CI: $6.0-8.1$ ) in 1991 to $13.00 \%$ (95\% CI: $11.1-15.1)$ in 2015. Prehypertension was consistently more prevalent in boys (than in girls) and in teenagers aged 13-17 years (than in those aged 7-12 years). For childhood hypertension, the overall agestandardized prevalence rose from $8.5 \%$ (95\% CI: 7.4-9.7) to 19.2\% (95\% CI: 16.9-21.7) across the same study time period, yielding a relative increasing rate of $5.3 \%(P<0.001)$. The prevalence of childhood hypertension was generally higher in participants aged 13-17 years (than in the younger groups). Besides, the age-standardized prevalence of prehypertension 
TABLE 3 | Characteristics of included subjects in CHNS 1991-2015.

\begin{tabular}{|c|c|c|c|c|c|c|c|c|c|c|}
\hline Characteristic & $\begin{array}{c}1991-2015 \text { combined } \\
(15,143)\end{array}$ & $1991(2,429)$ & $1993(2,254)$ & $1997(2,254)$ & $2000(2,217)$ & $2004(1,364)$ & $2006(1,145)$ & 20091,012 & $2011(1,404)$ & $2015(1,064)$ \\
\hline \multicolumn{11}{|l|}{ AGE GROUP } \\
\hline $7-12$ years & $8,783(58.00)$ & 1,335 (54.96) & $1,285(57.01)$ & $1,358(60.25)$ & $1,207(54.44)$ & $646(47.36)$ & $667(58.25)$ & $618(61.07)$ & $874(62.25)$ & $793(74.53)$ \\
\hline 13-17 years & $6,360(42.00)$ & 1,094 (45.04) & 969 (42.99) & $896(39.75)$ & $1,010(45.56)$ & $718(52.64)$ & $478(41.75)$ & $394(38.93)$ & $530(37.75)$ & $271(25.47)$ \\
\hline \multicolumn{11}{|l|}{ SEX } \\
\hline Male & $7,947(52.48)$ & $1,251(51.50)$ & $1,175(52.13)$ & $1,192(52.88)$ & $1,176(53.04)$ & $720(52.79)$ & 609 (53.19) & $562(55.53)$ & $713(50.78)$ & $549(51.60)$ \\
\hline Female & $7,196(47.52)$ & $1,178(48.50)$ & $1,079(47.87)$ & $1,062(47.12)$ & $1,041(46.96)$ & $644(47.21)$ & $536(46.81)$ & $450(44.47)$ & $691(49.22)$ & $515(48.40)$ \\
\hline \multicolumn{11}{|c|}{ ANTHROPOMETRIC MEASURES } \\
\hline Height, cm & $144.65 \pm 0.26$ & $142.37 \pm 0.64$ & $142.11 \pm 0.68$ & $142.62 \pm 0.65$ & $146.41 \pm 0.63$ & $149.05 \pm 0.86$ & $146.04 \pm 1.00$ & $146.44 \pm 0.99$ & $146.47 \pm 0.88$ & $144.55 \pm 0.97$ \\
\hline Weight, kg & $38.18 \pm 0.21$ & $36.39 \pm 0.49$ & $36.05 \pm 0.50$ & $36.55 \pm 0.50$ & $38.89 \pm 0.50$ & $41.33 \pm 0.69$ & $39.12 \pm 0.77$ & $39.29 \pm 0.82$ & $40.67 \pm 0.77$ & $39.35 \pm 0.96$ \\
\hline BMI, $\mathrm{kg} / \mathrm{m}^{2}$ & $17.69 \pm 0.05$ & $17.38 \pm 0.11$ & $17.28 \pm 0.12$ & $17.43 \pm 0.11$ & $17.68 \pm 0.11$ & $18.10 \pm 0.15$ & $17.77 \pm 0.18$ & $17.79 \pm 0.20$ & $18.35 \pm 0.20$ & $18.28 \pm 0.34$ \\
\hline wc, cm & $62.87 \pm 0.19$ & NA & $62.39 \pm 0.47$ & $61.39 \pm 0.35$ & $62.80 \pm 0.35$ & $64.10 \pm 0.50$ & $62.52 \pm 0.58$ & $63.07 \pm 0.62$ & $64.49 \pm 0.63$ & $63.03 \pm 0.83$ \\
\hline \multicolumn{11}{|c|}{ BLOOD PRESSURE MEASURES } \\
\hline $\mathrm{SBP}, \mathrm{mmHg}$ & $98.44 \pm 0.20$ & $96.18 \pm 0.53$ & $96.34 \pm 0.54$ & $97.18 \pm 0.53$ & $99.49 \pm 0.54$ & $101.53 \pm 0.70$ & $98.00 \pm 0.73$ & $99.83 \pm 0.78$ & $100.12 \pm 0.65$ & $101.54 \pm 0.76$ \\
\hline DBP, mmHg & $64.57 \pm 0.15$ & $62.61 \pm 0.39$ & $63.25 \pm 0.41$ & $63.67 \pm 0.40$ & $65.15 \pm 0.39$ & $66.66 \pm 0.51$ & $64.74 \pm 0.52$ & $66.63 \pm 0.57$ & $65.36 \pm 0.47$ & $66.65 \pm 0.55$ \\
\hline \multicolumn{11}{|l|}{ SETTING } \\
\hline Urban & $4,347(28.71)$ & $620(25.52)$ & $566(25.11)$ & 659 (29.24) & $609(27.47)$ & 408 (29.91) & $343(29.96)$ & 274 (27.08) & $530(37.75)$ & $338(31.77)$ \\
\hline Rural & $10,796(71.29)$ & $1,809(74.48)$ & 1,688 (74.89) & 1,595 (70.76) & 1,608 (72.53) & 956 (70.09) & 802 (70.04) & 738 (72.92) & 874 (62.25) & 726 (68.23) \\
\hline \multicolumn{11}{|l|}{ REGION } \\
\hline North & $5,286(34.91)$ & 728 (29.97) & 698 (30.97) & 776 (34.43) & 964 (43.48) & 556 (40.76) & 445 (38.86) & 375 (37.06) & 442 (31.48) & 302 (28.38) \\
\hline South & 9,857 (65.09) & 1,701 (70.03) & 1,556 (69.03) & $1,478(65.57)$ & 1,253 (56.52) & 808 (59.24) & $700(61.14)$ & 637 (62.94) & 962 (68.52) & 762 (71.62) \\
\hline
\end{tabular}

*Data are expressed as n (\%) or mean \pm SD; BMI, body mass index; WC, Waist circumference; SBP, systolic blood pressure; DBP, diastolic blood pressure. NA, Not available. 
TABLE 4 | Mean SBP and DBP in Chinese children and adolescents, CHNS 1991-2015.

\begin{tabular}{|c|c|c|c|c|c|c|c|c|c|c|c|c|}
\hline Variable & $\begin{array}{c}1991 \\
(n=2,429)\end{array}$ & $\begin{array}{c}1993 \\
(n=2,254)\end{array}$ & $\begin{array}{c}1997 \\
(n=2,254)\end{array}$ & $\begin{array}{c}2000 \\
(n=2,217)\end{array}$ & $\begin{array}{c}2004 \\
(n=1,364)\end{array}$ & $\begin{array}{c}2006 \\
(n=1,145)\end{array}$ & $\begin{array}{c}2009 \\
(n=1,012)\end{array}$ & $\begin{array}{c}2011 \\
(n=1,404)\end{array}$ & $\begin{array}{c}2015 \\
(n=10,64)\end{array}$ & $\begin{array}{l}\text { AAI } \\
\text { (\%) }\end{array}$ & $\begin{array}{l}\text { ARI } \\
(\%)\end{array}$ & $\begin{array}{c}P \text { for } \\
\text { age-adjusted } \\
\text { trend }\end{array}$ \\
\hline \multicolumn{13}{|c|}{ SBP $(\mathrm{mmHg})$, mean $(95 \% \mathrm{Cl})$} \\
\hline Overall & $\begin{array}{c}96.18 \\
(95.65-96.71)\end{array}$ & $\begin{array}{c}96.34 \\
(95.8-96.88)\end{array}$ & $\begin{array}{c}97.18 \\
(96.65-97.72)\end{array}$ & $\begin{array}{c}99.49 \\
(98.95-100.02)\end{array}$ & $\begin{array}{c}101.53 \\
(100.83-102.24)\end{array}$ & $\begin{array}{c}98.00 \\
(97.27-98.73)\end{array}$ & $\begin{array}{c}99.83 \\
(99.05-100.62)\end{array}$ & $\begin{array}{c}100.12 \\
(99.47-100.77)\end{array}$ & $\begin{array}{c}101.54 \\
(100.78-102.3)\end{array}$ & 0.22 & 0.23 & $<0.001$ \\
\hline \multicolumn{13}{|l|}{ AGE GROUP } \\
\hline $7-12$ years & $\begin{array}{c}90.47 \\
(89.88-91.06)\end{array}$ & $\begin{array}{c}91.59 \\
(90.93-92.25)\end{array}$ & $\begin{array}{c}92.65 \\
(92.02-93.27)\end{array}$ & $\begin{array}{c}95.14 \\
(94.48-95.8)\end{array}$ & $\begin{array}{c}95.27 \\
(94.32-96.21)\end{array}$ & $\begin{array}{c}92.98 \\
(92.12-93.85)\end{array}$ & $\begin{array}{c}96.54 \\
(95.54-97.55)\end{array}$ & $\begin{array}{c}96.24 \\
(95.47-97)\end{array}$ & $\begin{array}{c}99.23 \\
(98.37-100.09)\end{array}$ & 0.37 & 0.40 & $<0.001$ \\
\hline 13-17 years & $\begin{array}{c}103.15 \\
(102.4-103.9)\end{array}$ & $\begin{array}{c}102.64 \\
(101.91-103.37)\end{array}$ & $\begin{array}{c}104.05 \\
(103.29-104.82)\end{array}$ & $\begin{array}{c}104.68 \\
(103.92-105.44)\end{array}$ & $\begin{array}{c}107.17 \\
(106.34-108.01)\end{array}$ & $\begin{array}{c}105.00 \\
(104.02-105.97)\end{array}$ & $\begin{array}{c}104.99 \\
(103.93-106.06)\end{array}$ & $\begin{array}{c}106.52 \\
(105.57-107.46)\end{array}$ & $\begin{array}{c}108.29 \\
(106.94-109.65)\end{array}$ & 0.21 & 0.21 & $<0.001$ \\
\hline \multicolumn{13}{|l|}{ SEX } \\
\hline Male & $\begin{array}{c}96.51 \\
(95.76-97.26)\end{array}$ & $\begin{array}{c}96.67 \\
(95.88-97.45)\end{array}$ & $\begin{array}{c}97.19 \\
(96.45-97.93)\end{array}$ & $\begin{array}{c}100.32 \\
(99.56-101.09)\end{array}$ & $\begin{array}{c}102.48 \\
(101.47-103.48)\end{array}$ & $\begin{array}{c}98.76 \\
(97.71-99.81)\end{array}$ & $\begin{array}{c}100.20 \\
(99.11-101.29)\end{array}$ & $\begin{array}{c}101.24 \\
(100.29-102.19)\end{array}$ & $\begin{array}{c}102.90 \\
(101.81-103.99)\end{array}$ & 0.27 & 0.28 & $<0.001$ \\
\hline Female & $\begin{array}{c}95.83 \\
(95.08-96.58)\end{array}$ & $\begin{array}{c}95.99 \\
(95.26-96.72)\end{array}$ & $\begin{array}{c}97.17 \\
(96.39-97.95)\end{array}$ & $\begin{array}{c}98.54 \\
(97.8-99.29)\end{array}$ & $\begin{array}{c}100.48 \\
(99.52-101.45)\end{array}$ & $\begin{array}{c}97.13 \\
(96.12-98.15)\end{array}$ & $\begin{array}{c}99.37 \\
(98.25-100.5)\end{array}$ & $\begin{array}{c}98.96 \\
(98.09-99.84)\end{array}$ & $\begin{array}{c}100.09 \\
(99.03-101.14)\end{array}$ & 0.18 & 0.19 & $<0.001$ \\
\hline \multicolumn{13}{|c|}{ DBP $(\mathrm{mmHg})$, mean $(95 \% \mathrm{Cl})$} \\
\hline Overall & $\begin{array}{c}62.61 \\
(62.22-63.01)\end{array}$ & $\begin{array}{c}63.25 \\
(62.84-63.66)\end{array}$ & $\begin{array}{c}63.67 \\
(63.27-64.08)\end{array}$ & $\begin{array}{c}65.15 \\
(64.76-65.53)\end{array}$ & $\begin{array}{c}66.66 \\
(66.15-67.18)\end{array}$ & $\begin{array}{c}64.74 \\
(64.22-65.26)\end{array}$ & $\begin{array}{c}66.63 \\
(66.06-67.2)\end{array}$ & $\begin{array}{c}65.36 \\
(64.89-65.84)\end{array}$ & $\begin{array}{c}66.65 \\
(66.1-67.2)\end{array}$ & 0.17 & 0.27 & $<0.001$ \\
\hline \multicolumn{13}{|c|}{ AGE GROUP } \\
\hline $7-12$ years & $\begin{array}{c}58.99 \\
(58.5-59.47)\end{array}$ & $\begin{array}{c}60.23 \\
(59.7-60.76)\end{array}$ & $\begin{array}{c}60.77 \\
(60.28-61.27)\end{array}$ & $\begin{array}{c}62.49 \\
(61.99-62.99)\end{array}$ & $\begin{array}{c}63.09 \\
(62.35-63.83)\end{array}$ & $\begin{array}{c}61.52 \\
(60.91-62.12)\end{array}$ & $\begin{array}{c}64.77 \\
(64.04-65.5)\end{array}$ & $\begin{array}{c}62.95 \\
(62.38-63.52)\end{array}$ & $\begin{array}{c}65.49 \\
(64.86-66.11)\end{array}$ & 0.27 & 0.46 & $<0.001$ \\
\hline 13-17 years & $\begin{array}{c}67.04 \\
(66.5-67.58)\end{array}$ & $\begin{array}{c}67.26 \\
(66.7-67.82)\end{array}$ & $\begin{array}{c}68.07 \\
(67.49-68.65)\end{array}$ & $\begin{array}{c}68.33 \\
(67.78-68.87)\end{array}$ & $\begin{array}{c}69.88 \\
(69.26-70.5)\end{array}$ & $\begin{array}{c}69.24 \\
(68.48-70)\end{array}$ & $\begin{array}{c}69.55 \\
(68.71-70.38)\end{array}$ & $\begin{array}{c}98.96 \\
(68.64-70.07)\end{array}$ & $\begin{array}{c}100.09 \\
(68.97-71.09)\end{array}$ & 0.12 & 0.19 & $<0.001$ \\
\hline SEX & & & & & & & & & & & & \\
\hline Male & $\begin{array}{c}62.66 \\
(62.1-63.21)\end{array}$ & $\begin{array}{c}63.43 \\
(62.84-64.03)\end{array}$ & $\begin{array}{c}63.58 \\
(63.04-64.12)\end{array}$ & $\begin{array}{c}65.55 \\
(65.01-66.09)\end{array}$ & $\begin{array}{c}67.13 \\
(66.41-67.84)\end{array}$ & $\begin{array}{c}65.14 \\
(64.39-65.89)\end{array}$ & $\begin{array}{c}66.67 \\
(65.9-67.44)\end{array}$ & $\begin{array}{c}66.36 \\
(65.67-67.05)\end{array}$ & $\begin{array}{c}67.46 \\
(66.66-68.26)\end{array}$ & 0.20 & 0.32 & $<0.001$ \\
\hline Female & $\begin{array}{c}62.57 \\
(62.01-63.13)\end{array}$ & $\begin{array}{c}63.06 \\
(62.48-63.63)\end{array}$ & $\begin{array}{c}63.78 \\
(63.17-64.38)\end{array}$ & $\begin{array}{c}64.69 \\
(64.14-65.24)\end{array}$ & $\begin{array}{c}66.15 \\
(65.41-66.88)\end{array}$ & $\begin{array}{c}64.29 \\
(63.58-65.01)\end{array}$ & $\begin{array}{c}66.58 \\
(65.73-67.43)\end{array}$ & $\begin{array}{c}64.34 \\
(63.69-64.98)\end{array}$ & $\begin{array}{c}65.78 \\
(65.03-66.52)\end{array}$ & 0.13 & 0.21 & $<0.001$ \\
\hline
\end{tabular}

AAl, Average annual increase, AAI was computed by dividing the difference of blood pressure between 1991 and 2015 by the number of years covered; ARI = Average relative increase, ARI was calculated by dividing the average annual change by the baseline mean blood pressure in 1991; trends in mean blood pressure from 1991 to 2015 were assessed by the generalized-estimating-equation method and adjusted by age and sex. 
TABLE 5 | Prevalence of hypertension and phenotypes in Chinese children and adolescents, CHNS 1991-2015.

\begin{tabular}{|c|c|c|c|c|c|c|c|c|c|c|c|c|}
\hline Variable & $\begin{array}{c}1991 \\
(n=2,429)\end{array}$ & $\begin{array}{c}1993 \\
(n=2,254)\end{array}$ & $1997(n=2254)$ & $2000(n=2217)$ & $\begin{array}{c}2004 \\
(n=1,364)\end{array}$ & $\begin{array}{c}2006 \\
(n=1,145)\end{array}$ & $\begin{array}{c}2009 \\
(n=1,012)\end{array}$ & $2011(n=1404)$ & $\begin{array}{c}2015 \\
(n=1,064)\end{array}$ & $\begin{array}{l}\text { AAI } \\
\text { (\%) }\end{array}$ & $\begin{array}{l}\text { ARI } \\
\text { (\%) }\end{array}$ & $\begin{array}{c}P \text { for } \\
\text { age-adjusted } \\
\text { trend }\end{array}$ \\
\hline
\end{tabular}

Prehypertension $(\%, 95 \% \mathrm{Cl})$

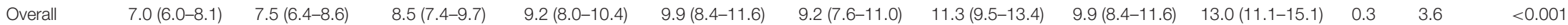

\section{AGE GROUP}

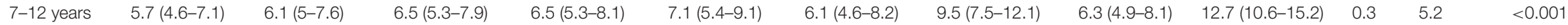

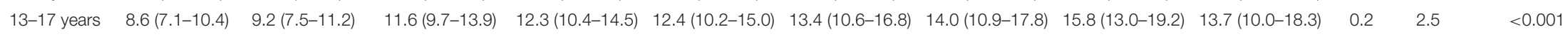

SEX

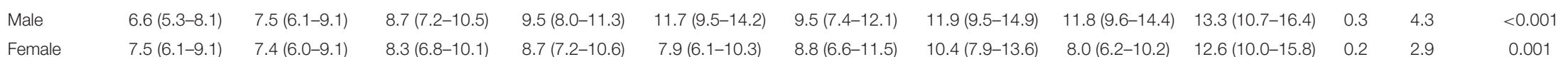

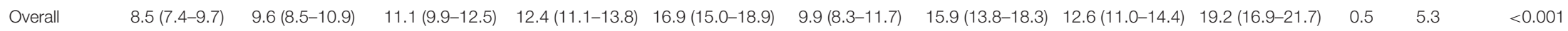

AGE GROUP

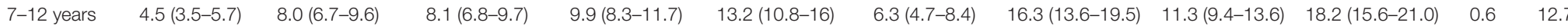

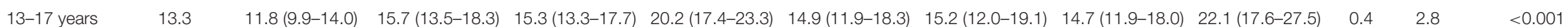

SEX

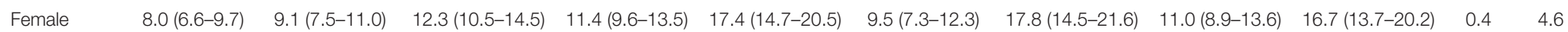

Stage 1 hypertension $(\%, 95 \% \mathrm{Cl})$

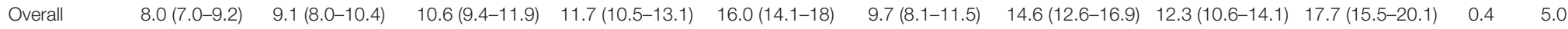

AGE GROUP

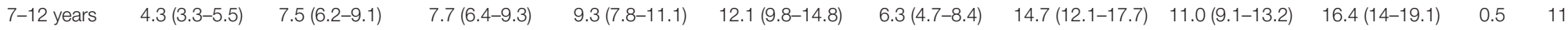

$11.1(0.3-13.3) \quad 14.8(12.7-17.3) 14.7(12.6-17.0) \quad 19.5(16.8-22.0) \quad 14.4(11.6-17.9)-14.5(11.3-18.3) 14.3(11.6-17.0) 21.4(16.9-26.7) \quad 0.4$ (10.8-14.7)

SEX

Male

(1)

Stage 2 hypertension (\%, 95\% Cl)

\begin{tabular}{|c|c|c|c|c|c|c|c|c|c|c|c|c|}
\hline Overall & $0.5(0.3-0.8)$ & $0.5(0.3-0.9)$ & $0.6(0.3-1.0)$ & $0.6(0.4-1.1)$ & $0.9(0.5-1.5)$ & $0.2(0.0-0.7)$ & $1.3(0.7-2.2)$ & $0.4(0.1-0.9)$ & $1.5(0.9-2.4)$ & 0.0 & 9.7 & 0.012 \\
\hline \multicolumn{13}{|l|}{ AGE GROUP } \\
\hline $7-12$ years & $0.2(0.1-0.7)$ & $0.5(0.2-1.0)$ & $0.4(0.2-0.9)$ & $0.6(0.3-1.2)$ & $1.1(0.5-2.3)$ & $0.0(0.0-0.0)$ & $1.6(0.9-3.0)$ & $0.3(0.1-1.1)$ & $1.8(1.0-3.0)$ & 0.06 & 28.6 & $<0.001$ \\
\hline $13-17$ years & $0.7(0.4-1.5)$ & $0.6(0.3-1.4)$ & $0.9(0.4-1.8)$ & $0.7(0.3-1.4)$ & $0.7(0.3-1.7)$ & $0.4(0.1-1.7)$ & $0.8(0.2-2.3)$ & $0.4(0.1-1.5)$ & $0.7(0.2-2.9)$ & 0.0 & 0.0 & 0.589 \\
\hline \multicolumn{13}{|l|}{ SEX } \\
\hline Male & $0.2(0.1-0.7)$ & $0.7(0.3-1.4)$ & $0.3(0.1-0.8)$ & $0.8(0.4-1.5)$ & $0.8(0.4-1.8)$ & $0.3(0.1-1.3)$ & $1.2(0.6-2.6)$ & $0.4(0.1-1.3)$ & $1.1(0.5-2.4)$ & 0.0 & 14.8 & 0.045 \\
\hline Female & $0.7(0.3-1.4)$ & $0.4(0.1-1.0)$ & $0.9(0.5-1.7)$ & $0.5(0.2-1.1)$ & $0.9(0.4-2.1)$ & $0.0(0.0-0.0)$ & $1.3(0.6-2.9)$ & $0.3(0.1-1.2)$ & $1.9(1.0-3.6)$ & 0.1 & 7.8 & 0.110 \\
\hline \multicolumn{13}{|c|}{ ISH (\%, 95\% CI) } \\
\hline Overall & $0.9(0.6-1.4)$ & $0.8(0.5-1.3)$ & $1.6(1.1-2.2)$ & $1.4(0.9-1.9)$ & $2.1(1.5-3.0)$ & $1.1(0.7-1.9)$ & $1.6(1.0-2.6)$ & $1.1(0.7-1.9)$ & $2.2(1.4-3.2)$ & 0.1 & 5.4 & 0.001 \\
\hline
\end{tabular}


TABLE 5 | Continued

\begin{tabular}{|c|c|c|c|c|c|c|c|c|c|c|c|c|}
\hline Variable & $\begin{array}{c}1991 \\
(n=2,429)\end{array}$ & $\begin{array}{c}1993 \\
(n=2,254)\end{array}$ & $1997(n=2254)$ & $2000(n=2217)$ & $\begin{array}{c}2004 \\
(n=1,364)\end{array}$ & $\begin{array}{c}2006 \\
(n=1,145)\end{array}$ & $\begin{array}{c}2009 \\
(n=1,012)\end{array}$ & $2011(n=1404)$ & $\begin{array}{c}2015 \\
(n=1,064)\end{array}$ & $\begin{array}{l}\text { AAI } \\
(\%)\end{array}$ & $\begin{array}{l}\text { ARI } \\
(\%)\end{array}$ & $\begin{array}{c}P \text { for } \\
\text { age-adjusted } \\
\text { trend }\end{array}$ \\
\hline \multicolumn{13}{|l|}{ AGE GROUP } \\
\hline $7-12$ years & $1(0.6-1.8)$ & $0.8(0.4-1.5)$ & $1.2(0.7-2.0)$ & $1.3(0.8-2.1)$ & $1.7(0.9-2.9)$ & $1.3(0.7-2.6)$ & $2.0(1.1-3.5)$ & $1.5(0.9-2.8)$ & $1.5(0.7-2.9)$ & 0.02 & 1.7 & $<0.001$ \\
\hline $13-17$ years & $0.8(0.5-1.6)$ & $0.8(0.4-1.6)$ & $2.0(1.3-3.0)$ & $1.4(0.9-2.4)$ & $2.6(1.6-4.2)$ & $0.9(0.4-2.2)$ & $1.1(0.5-2.6)$ & $0.7(0.3-1.7)$ & $2.9(1.8-4.8)$ & 0.1 & 10.1 & 0.932 \\
\hline \multicolumn{13}{|l|}{ SEX } \\
\hline Male & $0.3(0.1-0.8)$ & $0.5(0.3-1.1)$ & $1.0(0.6-1.7)$ & $1.3(0.8-2.2)$ & $1.1(0.5-2.3)$ & $1.2(0.6-2.4)$ & $1.9(1.1-3.4)$ & $1.3(0.7-2.3)$ & $2.0(1.2-3.3)$ & 0.1 & 23.9 & 0.027 \\
\hline Female & $1.7(1.1-2.7)$ & $1.1(0.6-2.0)$ & $2.3(1.5-3.6)$ & $1.4(0.8-2.3)$ & $3.1(2.0-4.6)$ & $1.0(0.4-2.5)$ & $1.0(0.4-2.7)$ & $0.9(0.4-2.2)$ & $2.6(1.2-5.3)$ & 0.0 & 2.0 & 0.021 \\
\hline \multicolumn{13}{|c|}{ IDH (\%, 95\% Cl) } \\
\hline Overall & $6.2(5.3-7.2)$ & $7.5(6.5-8.7)$ & $7.5(6.5-8.7)$ & $8.9(7.8-10.1)$ & $13.0(11.3-14.9)$ & $7.2(5.8-8.8)$ & $12.1(10.2-14.2)$ & $9.2(7.8-10.8)$ & $14.1(12.1-16.3)$ & 0.3 & 5.4 & $<0.001$ \\
\hline \multicolumn{13}{|l|}{ AGE GROUP } \\
\hline $7-12$ years & $3.6(2.7-4.7)$ & $6.1(4.9-7.5)$ & 4.9 (3.9-6.2) & $6.1(4.9-7.6)$ & $10.5(8.4-13.1)$ & $3.1(2.1-4.8)$ & 11.7 (9.3-14.4) & $7.8(6.2-9.8)$ & $12.6(10.5-15.1)$ & 0.4 & 10.5 & $<0.001$ \\
\hline $13-17$ years & $9.3(7.7-11.2)$ & $9.5(7.8-11.5)$ & $11.4(9.5-13.6)$ & $12.2(10.3-14.3)$ & $15.2(12.7-18)$ & $12.8(10.1-16.1)$ & $12.7(9.7-16.4)$ & $11.5(9.1-14.5)$ & $18.5(14.3-23.5)$ & 0.4 & 4.1 & $<0.001$ \\
\hline \multicolumn{13}{|l|}{ SEX } \\
\hline Male & $6.6(5.4-8.2)$ & $7.8(6.4-9.5)$ & 7.6 (6.3-9.3) & $9.7(8.1-11.5)$ & $12.8(10.5-15.4)$ & $7.4(5.6-9.8)$ & $10.5(8.2-13.3)$ & $10.2(8.2-12.7)$ & $16.4(13.5-19.7)$ & 0.4 & 6.1 & $<0.001$ \\
\hline Female & $5.7(4.5-7.2)$ & $7.2(5.8-8.9)$ & $7.3(5.9-9.1)$ & $8.0(6.5-9.8)$ & $13.2(10.8-16.0)$ & $6.9(5.0-9.4)$ & $14.0(11.1-17.5)$ & $8.1(6.3-10.4)$ & $11.7(9.1-14.7)$ & 0.3 & 4.4 & $<0.001$ \\
\hline \multicolumn{13}{|c|}{ SDH (\%, 95\% Cl) } \\
\hline Overall & $1.4(1.4-1.9)$ & $1.3(0.9-1.8)$ & $2.1(1.6-2.8)$ & $2.1(1.6-2.8)$ & $1.8(1.2-2.6)$ & $1.6(1.0-2.5)$ & $2.3(1.5-3.4)$ & $2.3(1.6-3.2)$ & $2.9(2.1-4.1)$ & 0.1 & 4.8 & 0.001 \\
\hline \multicolumn{13}{|l|}{ AGE GROUP } \\
\hline $7-12$ years & $0.6(0.3-1.2)$ & $1.4(0.9-2.2)$ & $2.1(1.5-3.1)$ & $2.4(1.7-3.4)$ & $1.5(0.8-2.9)$ & $1.9(1.1-3.3)$ & $2.8(1.7-4.4)$ & $2.3(1.5-3.5)$ & $3.5(2.4-5.1)$ & 0.12 & 20.4 & $<0.001$ \\
\hline $13-17$ years & $2.3(1.5-3.4)$ & $1.1(0.6-2.0)$ & $2.0(1.3-3.2)$ & $1.8(1.1-2.8)$ & $1.9(1.2-3.3)$ & $1.0(0.4-2.5)$ & $1.5(0.7-3.4)$ & $2.3(1.3-3.9)$ & $1.1(0.4-3.4)$ & -0.1 & -2.2 & 0.632 \\
\hline \multicolumn{13}{|l|}{ SEX } \\
\hline Male & $1.3(0.8-2.1)$ & $1.5(1.0-2.4)$ & $1.3(0.8-2.1)$ & $2.2(1.5-3.2)$ & $1.9(1.2-3.3)$ & $1.5(0.8-2.8)$ & $2.0(1.1-3.5)$ & $2.4(1.5-3.8)$ & $3.6(2.4-5.6)$ & 0.1 & 7.7 & 0.002 \\
\hline Female & $1.4(0.9-2.3)$ & $1.0(0.6-1.8)$ & $3.0(2.1-4.2)$ & $2.0(1.3-3.1)$ & $1.6(0.8-2.9)$ & $1.7(0.9-3.2)$ & $2.7(1.5-4.6)$ & $2.2(1.3-3.6)$ & $2.1(1.2-3.8)$ & 0.0 & 2.0 & 0.146 \\
\hline
\end{tabular}

AAl, Average annual increase, AAl was computed by dividing the difference of prevalence between 1991 and 2015 by the number of years covered; ARI=Average relative increase, ARI was calculated by dividing the average annual change by the baseline prevalence in 1991; trends in prevalence from 1991 to 2015 were assessed by the generalized-estimating-equation method and adjusted by age and sex. 


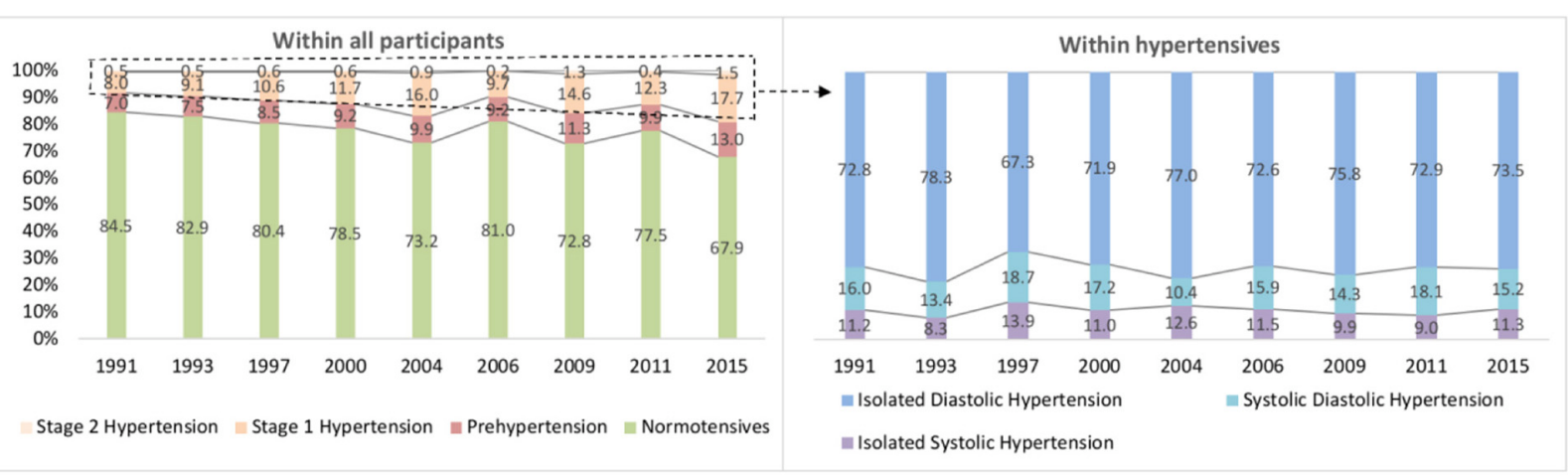

FIGURE 1 | The proportions of childhood hypertension and phenotypes, CHNS 1991-2015.

and hypertension increased in all age and sex subgroups $(P<0.001)$ and the prevalence of hypertension in children aged 7-12 years increased the most during the survey rounds (increased by $12.7 \%$ ).

For stage 1 and stage 2 hypertension, the age-standardized prevalence increased from $8.0 \%$ (95\% CI: 7.0-9.2) to $17.7 \%(95 \%$ CI: $15.5-20.1)$ and from $0.5 \%$ (95\% CI: $0.3-0.8)$ to $1.5 \%$ (95\% CI: $0.9-2.4)$, respectively. The prevalence of stage 1 hypertension was generally higher than that of stage 2 hypertension. Within this time frame, the ARI rates of both stage 1 and stage 2 hypertension were greater in $7-12$ years old children (increased by $11.8 \%$ and $28.6 \%$ ) than in older groups (increased by $2.9 \%$ and $0.0 \%$ ). No statistically significant prevalence trend in stage 2 hypertension was observed among people aged 13-17 years and in girls $(P=$ 0.589 and 0.110 , separately).

IDH accounted for the largest share of hypertension phenotypes (Figure 1). For ISH, the age-standardized prevalence increased significantly from $0.9 \%$ (95\% CI: $0.6-1.4)$ to $2.2 \%(95 \%$ CI: 1.4-3.2) from CHNS 1991 to CHNS 2015. Upward trends were observed in all subgroups (males, females and 7-12 years), except in people aged $13-17(P=0.932)$. For IDH, the agestandardized prevalence increased from $6.2 \%$ to $14.1 \%$ (boys from 6.6 to $16.4 \%$, girls from 5.7 to $11.7 \%$ ) over 25 years. The prevalence of IDH in two age groups (7-12 and 13-17 years) increased by $10.5 \%$ and $4.1 \%$ (both age-adjusted $P<0.001$ ). The age-standardized prevalence of SDH increased from $1.4 \%$ (95\% CI: 1.4-1.9) in 1991 to 2.9\% (95\% CI: 2.1-4.1) in 2015. An increasing trend of SDH prevalence was revealed in the age group of 7-12 years (from 0.6 to $3.5 \%$ ) and in boys (from 1.3 to $3.6 \%$ ) (Table 5).

\section{The Associations of Demographic, Geographic, Anthropometric Factors, and Obesity With Hypertension and Hypertension Phenotypes}

Of the 15,143 records, 11,450 were with complete information on BP, weight, height, WC, HC, age, sex, setting and region. The demographic characteristics between the included $(n=15,143)$ group and excluded $(n=3,460)$ group were compared (Table 1). Of note, the included participants were relatively younger than the excluded individuals across all survey rounds $(P<0.05)$, except for in CHNS 2004 and 2011, where no sex difference was observed between the two groups (all $P>0.05$ ).

Table 6 shows the associated demographic, geographic and anthropometric factors of hypertension and phenotypes. Age, region, $\mathrm{BMI}, \mathrm{WC}$, general obesity, and central obesity were found to be associated with hypertension $(P<0.001)$. Compared with children aged 7-12 years, those aged older than 13 years and above were more likely to suffer from prehypertension $(\mathrm{OR}=$ 2.08, 95\%CI: 1.82-2.37, $P<0.001$ ) and stage 1 hypertension $(\mathrm{OR}=1.81,95 \% \mathrm{CI}: 1.61-2.03, P<0.001)$. Girls (vs. boys) were less likely to be affected by prehypertension $(\mathrm{OR}=0.83$, 95\%CI: $0.73-0.95, P=0.005)$, whereas no sex difference was observed in the prevalence of stage 1 and 2 hypertension. BMI and WC were significantly correlated with the prevalence of different hypertension phenotypes (all ORs with 95\%CI were $>1$ and $P<0.05$, except for the SDH). For prehypertension, stage 1 hypertension and stage 2 hypertension, general obesity was a positive factor, with ORs of 2.00 (95\%CI: 1.47-2.73, $P<$ 0.001 ), 2.62 (95\%CI: $2.03-3.38, P<0.001$ ), and 3.68 (95\%CI: $1.82-7.44, P<0.001)$, respectively. Central obesity was linked to both stages of hypertension additionally $(\mathrm{OR}=1.43,95 \% \mathrm{CI}$ : 1.16-1.78, $P=0.001$ and 2.76, 95\%CI: $1.48-5.14,0.001)$. For $\mathrm{IDH}$, advanced age (OR $=2.02,95 \% \mathrm{CI}: 1.77-2.31, P<0.001)$, general obesity $(\mathrm{OR}=1.83,95 \% \mathrm{CI}: 1.36-2.47, P<0.001)$ and central obesity $(\mathrm{OR}=1.50,95 \% \mathrm{CI}: 1.19-1.90, P=0.001)$ were positively associated with higher odds, whereas females, rural residents and south region were negative factors $(\mathrm{ORs}=0.86,0.86$ and 0.59 , all $P<0.05$ ). Similar to IDH, three out of these factors (13-17 years age group, general obesity and central obesity) were related to the increased prevalence of ISH $(\mathrm{ORs}=1.57,3.56$, and 2.43 , all $P<0.05)$. However, only general obesity contributed to the elevated prevalence of SDH, with an OR of 7.27 (95\%CI: 4.57-11.59, $P<0.001)$.

\section{DISCUSSION}

The current study describes the prevalence and associated factors of hypertension and phenotypes in Chinese children and adolescents during 1991-2015. It provides novel insights 
TABLE 6 | Multivariable odds ratios of demographic, geographic, and anthropometric factors and obesity for childhood hypertension and phenotypes.

\begin{tabular}{|c|c|c|c|c|c|c|c|c|c|c|c|c|c|c|}
\hline \multirow[t]{2}{*}{ Characteristic } & \multicolumn{2}{|c|}{ Prehypertension } & \multicolumn{2}{|c|}{ Hypertension } & \multicolumn{2}{|c|}{ Stage 1 hypertension } & \multicolumn{2}{|c|}{ Stage 2 hypertension } & \multicolumn{2}{|c|}{ ISH } & \multicolumn{2}{|c|}{ IDH } & \multicolumn{2}{|c|}{ SDH } \\
\hline & OR $(95 \% \mathrm{Cl})$ & $P$ & OR $(95 \% \mathrm{Cl})$ & $P$ & OR $(95 \% \mathrm{Cl})$ & $P$ & OR $(95 \% \mathrm{Cl})$ & $P$ & OR $(95 \% \mathrm{Cl})$ & $\boldsymbol{P}$ & OR $(95 \% \mathrm{Cl})$ & $P$ & OR $(95 \% \mathrm{Cl})$ & $P$ \\
\hline
\end{tabular}

\section{SURVEY YEAR}

1993

1997

$1 \quad$ Ref

\begin{tabular}{|c|c|c|c|c|c|}
\hline 1 & Ref & 1 & Ref & 1 & Ref \\
\hline $91-1.52)$ & 0.22 & $1.09(0.86-1.3$ & 0.484 & $1.11(0.88-1.41)$ & 0.380 \\
\hline
\end{tabular}

$1.17(0.91-1.52) \quad 0.226$

$\begin{array}{ccccccccccc}1.23(0.96-1.59) & 0.106 & 1.17(0.93-1.46) & 0.188 & 1.20(0.95-1.51) & 0.133 & 0.75(0.31-1.82) & 0.530 & 1.78(0.86-3.68) & 0.118 \\ 1.33(1.01-1.75) & 0.043 & 1.61(1.27-2.04) & <0.001 & 1.65(1.30-2.10) & <0.001 & 1.00(0.39-2.54) & 1.000 & 2.73(1.30-5.71) & 0.008\end{array}$

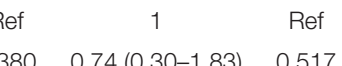

$1 \quad$ Ref

$\begin{array}{cc}1 & \text { Ref } \\ 0.97(0.74-1.26) & 0.800\end{array}$

1

2000

2006

2009

$\begin{array}{llllllllllll}1.61(1.21-2.16) & 0.001 & 1.60(1.24-2.07) & <0.001 & 1.60(1.23-2.08) & 0.001 & 1.57(0.67-3.68) & 0.296 & 2.03(0.93-4.45) & 0.076 & 1.60(1.20-2.13) & 0.001\end{array}$

2011

$\begin{array}{lllllll}1.35(1.02-1.78) & 0.035 & 1.12(0.87-1.44) & 0.377 & 1.18(0.92-1.53) & 0.192 & 0.33(0.11-1.05)\end{array}$

AGE GROUP

$7-12$ years

$2.14(1.62-2.85) \quad<0.001 \quad 2.08(1.61-2.67) \quad<0.001 \quad 2.09(1.61-2.71) \quad<0.001 \quad 1.53(0.65-3.59) \quad 0.327$

$\begin{array}{cccccccccccccc}1 & \text { Ref } & 1 & \text { Ref } & 1 & \text { Ref } & 1 & \text { Ref } & 1 & \text { Ref } & 1 & \text { Ref } & 1 & \text { Ref }\end{array}$

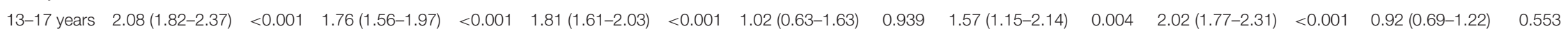

SEX

$\begin{array}{cccccccccccccc}\text { Male } & 1 & \text { Ref } & 1 & \text { Ref } & 1 & \text { Ref } & 1 & \text { Ref } & 1 & \text { Ref } & 1 & \text { Ref }\end{array}$

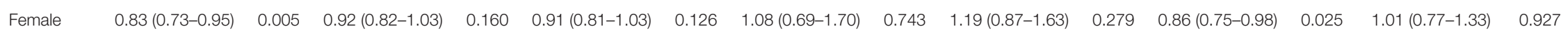

LOCATION

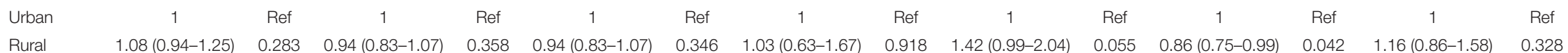

\section{REGION}

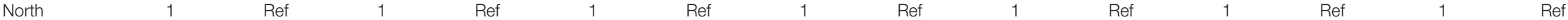

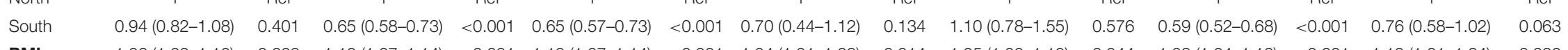

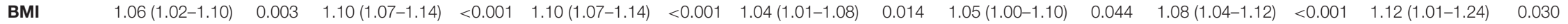

$\left(\mathrm{kg} / \mathrm{m}^{2}\right)$

WC (cm) $\quad 1.02(1.01-1.03) \quad<0.001 \quad 1.02(1.01-1.03) \quad<0.001 \quad 1.02(1.01-1.03) \quad<0.001 \quad 1.03(1.01-1.06) \quad 0.012 \quad 1.04(1.02-1.06) \quad<0.001 \quad 1.02(1.01-1.03) \quad<0.001 \quad 1.00(0.98-1.02) \quad 0.804$ GENERAL OBESITY

$\begin{array}{llllllllllll}\text { Normal } & 1 & \text { Ref } & 1 & \text { Ref } & 1 & \text { Ref } & 1 & \text { Ref } & 1 & \text { Ref }\end{array}$

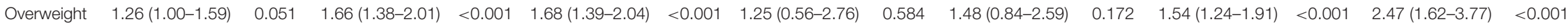

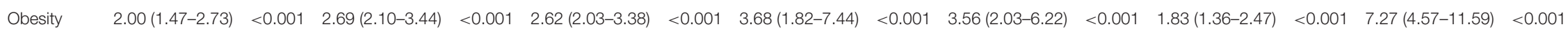
CENTRAL OBESITY

$\begin{array}{cccccccccccc}\text { Normal } & 1 & \text { Ref } & 1 & \text { Ref } & 1 & \text { Ref } & 1 & \text { Ref } & 1 & \text { Ref } & 1\end{array}$

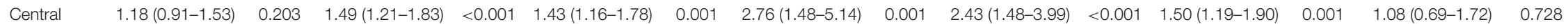

*Values were odds ratio (95\% confidence interval); Odds ratio for each variable was adjusted for all other variables in the table; Ref, reference; BMI, Body Mass Index; WC, Waist Circumference;The comparisons were between hypertension groups and normotensives; The multivariable generalized estimating equations (GEE) logistic regression adjusted all variables listed. 
into several aspects of the national challenge of childhood hypertension in China. From 1991 to 2015, the age-standardized prevalence of hypertension and phenotypes in Chinese children and adolescents increased dramatically. Sex and age disparities were found in the prevalence of childhood hypertension and phenotypes. For adolescents aged 13-17 years, general obesity and central obesity were positively associated with hypertension, whereas the South region was a negatively related factor. For IDH, sex, age, location (urban or rural), region (north or south), BMI, WC, general obesity and central obesity were associated factors. Older age group, general obesity and central obesity were related to the increased prevalence of ISH, and only general obesity associate with the elevated prevalence of IDH.

It is well-known that hypertension in children is associated with long-term adverse health effects (7). However, few studies reported the trends and related factors of childhood hypertension phenotypes. To the best of our knowledge, this study is the first to report the secular trends in the prevalence of hypertension phenotypes in such a large sample of Chinese children over more than two decades. Based on the stringent sampling approach and standardized procedures for BP measurements in CHNS, the quality of our results was largely ensured. In line with a national survey in the United States, significant increasing temporal trends in childhood BP and the prevalence of hypertension (from 8.5 to $19.2 \%$ ) were revealed in our study (29). However, such an upward secular trend was not universally observed in some previous studies due to the heterogeneity in demographic characteristics and socioeconomic status in different settings. For example, the Youth Heart Project in Northern Ireland has reported that the DBP has decreased by about ten $\mathrm{mmHg}$ during the past decade (30). In addition, a survey-based study in the United States showed that both mean SBP and DBP declined by 0.7 and $4.3 \mathrm{mmHg}$ among 17 -year-old adolescents from 1999-2002 to 2009-2012 (31). Liang et al. successfully revealed increasing trends in the prevalence of prehypertension and hypertension in Chinese children and adolescents from 1991 to 2004 (32). Compared with their study, we extended the study period from 1991-2004 to 1991-2015, additionally assessed the prevalence of hypertension according to severity (stage 1 hypertension and stage 2 hypertension) and phenotype (ISH, IDH, and SDH), and explored the effects of demographic, geographic factors, and obesity on hypertension.

IDH plays a critical role in childhood hypertension, and the prevalence was estimated to be $6.2-14.1 \%$ in this study, accounting for $67.3-78.3 \%$ of all phenotypes of hypertension. IDH in children and adolescents was defined as a DBP $\geq 95$ th percentile and an SBP $<$ 95th percentile for each age, sex, and height subgroup (10). It has been discovered that DBP levels were regulated by the renin-angiotensin-aldosterone system (RAAs) and vasoactive substances (33). Disease and unhealthy lifestyles may affect the regulation of RAAs and lead to a rise in DBP values (34-36).

Our results showed that the prevalence of hypertension and its phenotypes by sex and age subgroups increased dramatically over the recent two decades. Compared with girls, boys were more vulnerable to prehypertension and IDH. The sex differences in hypertension prevalence have been generally recognized as a result of hormones occurring at puberty (37). It has been reported that estrogen was essential for the enhancement of endotheliumdependent vasodilation and regulation of smooth muscle cells $(37,38)$. Besides, unhealthy behaviors, such as alcohol intake, sedentary lifestyle and obesity were more common in boys than girls in China, which may contribute to a greater risk of hypertension $(39,40)$. In the current study, the prevalence of hypertension among teenagers aged 13-17 years (ranging from 11.8 to $22.1 \%$ ) was higher than that in $7-12$ years old children (ranging from 4.5 to $18.2 \%$ ). According to clinical studies and animal experiments, the changes in vascular elasticity as children grow may partly explain the age disparities in the prevalence of hypertension among children $(41,42)$. Moreover, the prevalence of childhood hypertension differed in regions and locations. In comparison with urban children, rural children had a lower prevalence of IDH, which may be resulted from a better lifestyle and environment in rural settings, including more healthy eating patterns, more physical activities and fewer air pollutions $(43,44)$. Similarly, children living in South China were less likely to be affected by hypertension, stage 1 hypertension and IDH. It has been estimated that diary sodium intake in North China is higher than that in South China, which might have attributed to the differences across geographic regions (45).

It is widely acknowledged that obesity is one of the most important factors responsible for elevated BP in children and adolescents $(46,47)$. In accordance with a population-based study in Nigeria, where BMI and WC were revealed as risk factors for hypertension and prehypertension, we found that both general obesity and central obesity were associated factors of childhood hypertension (48). Previous studies also stated that the proportion of obese children suffering from hypertension was three times higher than that of non-obese children $(49,50)$. However, the mechanisms by which obesity directly influence BP is still unclear. It is reported that sympathicotonia and insulin resistance could regulate $\mathrm{BP}$ level through changing vascular reactivity and reduced-sodium excretion $(51,52)$. Besides, the accumulation of visceral adipose tissue triggers an immune response, resulting in an excessive amount of free fatty acids (FFA) (53). The high concentrated FFA in portal vein circulation inhibits the clearance of insulin and activates the production of angiotensinogen. In addition, the unbalanced angiotensinogen affects vasoconstriction and sodium reabsorption and, therefore, increases BP and develops hypertension (53).

\section{Limitations}

Several limitations should be considered when interpreting the results of this study. First, the national representativeness of CHNS could not be fully ensured, despite the large sample size and a wide range of economic and demographic variations. Second, BP was measured three times on a one-time visit rather than on three different occasions. The prevalence of hypertension might be overestimated (54). Third, people that were included at each study interval were not necessarily the same people, so the change in blood pressure over time may be related to sampling in each round. Fourth, several confounding factors, such as family economic level, family parent education, children's dietary patterns, comorbidities and medications were 
not accounted for in our multivariable analyses due to the absence of relative information.

\section{CONCLUSION}

In conclusion, BP levels and prevalence of hypertension and phenotypes increased dramatically in Chinese children and adolescents from 1991 to 2015. Regional discrepancy, demographic features, BMI, WC, and overweight/obesity status were associated factors of hypertension among youths. Our findings call for actions to identify and prevent childhood hypertension in China.

\section{DATA AVAILABILITY STATEMENT}

The datasets presented in this study can be found in online repositories. The names of the repository/repositories

\section{REFERENCES}

1. Kokubo Y, Matsumoto C. Hypertension is a risk factor for several types of heart disease: review of prospective studies. Adv Exp Med Biol. (2017) 956:419-26. doi: 10.1007/5584_2016_99

2. Girndt M. Diagnostik und Therapie der chronischen Nierenerkrankung. Der Internist. (2017) 58:243-56. doi: 10.1007/s00108-017-0195-2

3. Griffin G. Antiplatelet therapy and anticoagulation in patients with hypertension. Am Fam Physician. (2005) 71:8979. doi: 10.1001/jama.293.9.1123

4. Xiong X, Yang X, Liu Y, Zhang Y, Wang P, Wang J. Chinese herbal formulas for treating hypertension in traditional Chinese medicine: perspective of modern science. Hypertens Res. (2013) 36:570-9. doi: 10.1038/hr.2013.18

5. Center N C. China Cardiovascular Disease Report 2015. First Edition Ed. Beijing: China Encyclopedia press (2016). p. 13-24.

6. Song P, Zhang Y, Yu J, Zha M, Zhu Y, Rahimi K, et al. Global prevalence of hypertension in children. Jama Pediatr. (2019) 173:1154. doi: 10.1001/jamapediatrics.2019.3310

7. Rapsomaniki E, Timmis A, George J, Pujades-Rodriguez M, Shah $\mathrm{AD}$, Denaxas $\mathrm{S}$, et al. Blood pressure and incidence of twelve cardiovascular diseases: lifetime risks, healthy life-years lost, and age-specific associations in 1.25 million people. Lancet. (2014) 383:1899-911. doi: 10.1016/S0140-6736(14)60685-1

8. Hansen ML, Gunn PW, Kaelber DC. Underdiagnosis of hypertension in children and adolescents. JAMA. (2007) 298:874. doi: 10.1001/jama.298.8.874

9. Battistoni A, Canichella F, Pignatelli G, Ferrucci A, Tocci G, Volpe M. Hypertension in young people: epidemiology, diagnostic assessment and therapeutic approach. High Blood Pressur Cardiovasc Prev. (2015) 22:3818. doi: 10.1007/s40292-015-0114-3

10. Adolescents NHBP. The fourth report on the diagnosis, evaluation, and treatment of high blood pressure in children and adolescents. Pediatrics. (2004) 114:555-76. doi: 10.1161/01.HYP.0000143545.54637.af

11. Flynn JT, Kaelber DC, Baker-Smith CM, Blowey D, Carroll AE, Daniels $\mathrm{SR}$, et al. Clinical practice guideline for screening and management of high blood pressure in children and adolescents. Pediatrics. (2017) 140:e20171904. doi: 10.1542/peds.2017-1904

12. Burgner DP, Sabin MA, Magnussen CG, Cheung M, Sun C, Kähönen $M$, et al. Early childhood hospitalisation with infection and subclinical atherosclerosis in adulthood: The Cardiovascular Risk in Young Finns Study. Atherosclerosis. (2015) 239:496-502. doi: 10.1016/j.atherosclerosis.2015. 02.024

13. Beckett LA, Rosner B, Roche AF, Guo S. Serial changes in blood pressure from adolescence into adulthood. Am J Epidemiol. (1992) 135:116677. doi: 10.1093/oxfordjournals.aje.a116217 and accession number(s) can be found in the article/Supplementary Material.

\section{AUTHOR CONTRIBUTIONS}

PS designed the study. XY, QY, WX, and PS managed and analyzed the data. XY and QY prepared the first draft. All authors were involved in revising the paper and gave final approval of the submitted versions.

\section{SUPPLEMENTARY MATERIAL}

The Supplementary Material for this article can be found online at: https://www.frontiersin.org/articles/10.3389/fcvm. 2021.627741/full\#supplementary-material

14. Zhang Y, Wang S. Comparison of blood pressure levels among children and adolescents with different body mass index and waist circumference: study in a large sample in Shandong, China. Eur J Nutr. (2014) 53:62734. doi: 10.1007/s00394-013-0571-1

15. Marcovecchio M L, Mohn A, Diddi G, Polidori N, Chiarelli F, Fuiano N. Longitudinal assessment of blood pressure in schoolaged children: a 3-year follow-up study. Pediatr Cardiol. (2016) 37:255-61. doi: 10.1007/s00246-015-1271-9

16. Chiolero A, Cachat F, Burnier M, Paccaud F, Bovet P. Prevalence of hypertension in schoolchildren based on repeated measurements and association with overweight. J Hypertens. (2007) 25:220917. doi: 10.1097/HJH.0b013e3282ef48b2

17. Olsen MH, Angell SY, Asma S, Boutouyrie P, Burger D, Chirinos J A, et al. A call to action and a lifecourse strategy to address the global burden of raised blood pressure on current and future generations: the Lancet Commission on hypertension. Lancet. (2016) 388:2665712. doi: 10.1016/s0140-6736(16)31134-5

18. Popkin BM, Du S, Zhai F. Cohort profile: the china health and nutrition survey-monitoring and understanding socio-economic and health change in China, 1989-2011. Int J Epidemiol. (2010) 39:143540. doi: 10.1093/ije/dyp322

19. Zhang B, Zhai FY, Du SF, Popkin BM. The China health and nutrition survey, 1989-2011. Obes Rev. (2014) 15:2-7. doi: 10.1111/obr.12119

20. Organization WH. Physical status: the use and interpretation of anthropometry. Report of a WHO Expert Committee. WHO. (1995) 854:1452. doi: 10.1002/(sici)1520-6300(1996) 8:6<786:aid-ajhb11>3.0.co;2-i

21. Hui F, Yin-kun Y, Jie M. Establishing the user-friendly screening criteria for elevated blood pressure in Chinese children aged 3-17 years. Chinese J Hypert. (2017) 5:436-40. doi: 10.16439/j.cnki.1673-7245.2017.05.010

22. Sinha R, Saha A, Samuels J. American academy of pediatrics clinical practice guidelines for screening and management of high blood pressure in children and adolescents: what is new? Indian Pediatr. (2019) 56:31721. doi: $10.1007 / \mathrm{s} 13312-019-1523-5$

23. Xi B, Liang Y, He T, Reilly $\mathrm{KH}, \mathrm{Hu} \mathrm{Y}$, Wang Q, et al. Secular trends in the prevalence of general and abdominal obesity among Chinese adults, 1993-2009. Obes Rev. (2012) 13:287-96. doi: 10.1111/j.1467-789X.2011. 00944.x

24. Song $\mathrm{P}, \mathrm{Li} \mathrm{X}, \mathrm{Bu} \mathrm{Y}$, Ding $\mathrm{S}$, Zhai $\mathrm{D}$, Wang $\mathrm{E}$, et al. Temporal trends in normal weight central obesity and its associations with cardiometabolic risk among Chinese adults. Sci Rep-Uk. (2019) 9:5411. doi: 10.1038/s41598-01941986-5

25. Fan H, Yan Y, Mi J. Updating blood pressure references for Chinese children aged 3-17 years. Chinese J Hypert. (2017) 25:428-35. doi: 10.16439/j.cnki.1673-7245.2017.05.009 
26. Commission NHAF. Screening Criteria for Overweight and Obesity in SchoolAge Children and Adolescents. WS /T 586--2017. (2018). Available online at: http://ty.sx.gov.cn/art/2018/5/17/art_1489227_18292267.html.

27. Guerra MW, Shults J, Amsterdam J, Ten-Have T. The analysis of binary longitudinal data with time-dependent covariates. Stats Med. (2012) 31:93148. doi: $10.1002 / \operatorname{sim} .4465$

28. Zeger LSL. Longitudinal data analysis using generalized linear models. Biometrika. (1986) 73:13-22. doi: 10.1093/biomet/73.1.13

29. Din-Dzietham R, Liu Y, Bielo M, Shamsa F. High blood pressure trends in children and adolescents in national surveys, 1963 to 2002. Circulation. (2007) 116:1488-96. doi: 10.1161/CIRCULATIONAHA.106.683243

30. Watkins D, McCarron P, Murray L, Cran G, Boreham C, Robson P, et al. Trends in blood pressure over 10 years in adolescents: analyses of cross sectional surveys in the Northern Ireland Young Hearts project. BMJ. (2004) 329:139. doi: 10.1136/bmj.38149.510139.7C

31. Xi B, Zhang T, Zhang M, Liu F, Zong X, Zhao M, et al. Trends in elevated blood pressure among us children and adolescents: 1999-2012. Am J Hypertens. (2016) 29:217-25. doi: 10.1093/ajh/hpv091

32. Liang Y, Xi B, Hu Y, Wang C, Liu J, Yan Y, et al. Trends in blood pressure and hypertension among Chinese children and adolescents: China health and nutrition surveys 1991-2004. Blood Press. (2011) 20:4553. doi: 10.3109/08037051.2010.524085

33. Mattson D L. Immune mechanisms of salt-sensitive hypertension and renal end-organ damage. Nat Rev Nephrol. (2019) 15:290300. doi: 10.1038/s41581-019-0121-z

34. Franklin S S, Pio J R, Wong N D, Larson M G, Leip E P, Vasan R $\mathrm{S}$, et al. Predictors of new-onset diastolic and systolic hypertension. Circulation. (2005) 111:1121-7. doi: 10.1161/01.CIR.0000157159. 39889.EC

35. Gu M, Qi Y, Li M, Niu W. Association of body mass index and alcohol intake with hypertension subtypes among HAN chinese. Clin Exp Hypertens. (2011) 33:518-24. doi: 10.3109/10641963.2011.561899

36. Price A J, Crampin A C, Amberbir A, Kayuni-Chihana N, Musicha C, Tafatatha T, et al. Prevalence of obesity, hypertension, and diabetes, and cascade of care in sub-Saharan Africa: a cross-sectional, population-based study in rural and urban Malawi. Lancet Diabetes Endocri. (2018) 6:20822. doi: 10.1016/S2213-8587(17)30432-1

37. White K, Johansen AK, Nilsen M, Ciuclan L, Wallace E, Paton L, et al. Activity of the estrogen-metabolizing enzyme cytochrome p450 $1 \mathrm{~b} 1$ influences the development of pulmonary arterial hypertension. Circulation. (2012) 126:1087-98. doi: 10.1161/CIRCULATIONAHA.111.0 62927

38. Zhu Y. Abnormal vascular function and hypertension in mice deficient in estrogen receptor beta. Science. (2002) 295:5058. doi: 10.1126/science. 1065250

39. Du T, Sun X, Yin P, Yuan G, Zhang M, Zhou X, et al. Secular trends in the prevalence of low risk factor burden for cardiovascular disease according to obesity status among Chinese adults, 1993-2009. Bmc Public Health. (2014) 14:961. doi: 10.1186/1471-2458-14-961

40. Millwood IY, Li L, Smith M, Guo Y, Yang L, Bian Z, et al. Alcohol consumption in 0.5 million people from 10 diverse regions of China: prevalence, patterns and socio-demographic and health-related correlates. Int J Epidemiol. (2017) 46:2103. doi: 10.1093/ije/dyx210

41. Amin-Hanjani S, Du X, Pandey D K, Thulborn K R, Charbel F T. Effect of age and vascular anatomy on blood flow in major cerebral vessels. J Cerebral Blood Flow Metab. (2015) 35:312-8. doi: 10.1038/jcbfm. 2014.203
42. Sletner L, Mahon $\mathrm{P}$, Crozier $\mathrm{S} \mathrm{R}$, Inskip $\mathrm{H} \mathrm{M}$, Godfrey $\mathrm{K} \mathrm{M}$, Chiesa S, et al. Childhood fat and lean mass. Arterioscl Thromb Vasc Biol. (2018) 38:2528-37. doi: 10.1161/ATVBAHA.118. 311455

43. Fallah Z, Qorbani M, Motlagh ME, Heshmat R, Ardalan G, Kelishadi R. Prevalence of prehypertension and hypertension in a nationally representative sample of iranian children and adolescents: the CASPIAN-IV study. Int J Prev Med. (2014) 5 (Suppl. 1):S57-64.

44. Agyemang C, Redekop WK, Owusu-Dabo E, Bruijnzeels MA. Blood pressure patterns in rural, semi-urban and urban children in the Ashanti region of Ghana, West Africa. BMC Public Health. (2005) 5:114. doi: 10.1186/1471-2458-5-114

45. Huang L, Wang H, Wang Z, Wang Y, Zhang B, Ding G. Associations of dietary sodium, potassium, and sodium to potassium ratio with blood pressure-regional disparities in China. Nutrients. (2020) 12:366. doi: 10.3390/nu12020366

46. Moser DC, Giuliano IDCB, Titski ACK, Gaya AR, Coelho-e-Silva MJ, Leite N. Anthropometric measures and blood pressure in school children. J PediatBrazil. (2013) 89:243-9. doi: 10.1016/j.jped.2012.11.006

47. Ramesh V, Saraswat S, Choudhury N, Gupta RK. Relationship of serum alanine aminotransferase (ALT) to body mass index (BMI) in blood donors: the need to correct ALT for BMI in blood donor screening. Transfusion Med. (1995) 5:273-4. doi: 10.1111/j.1365-3148.1995.tb00213.x

48. Ononamadu CJ, Ezekwesili CN, Onyeukwu OF, Umeoguaju UF, Ezeigwe OC, Ihegboro GO. Comparative analysis of anthropometric indices of obesity as correlates and potential predictors of risk for hypertension and prehypertension in a population in Nigeria. Cardiovasc J Afr. (2017) 28:929. doi: 10.5830/CVJA-2016-061

49. Sorof JM, Poffenbarger T, Franco K, Bernard L, Portman RJ. Isolated systolic hypertension, obesity, and hyperkinetic hemodynamic states in children. $J$ Pediatrics. (2002) 140:660-6. doi: 10.1067/mpd.2002.125228

50. Sorof J, Daniels S. Obesity hypertension in children: a problem of epidemic proportions. Hypertension. (2002) 40:4417. doi: 10.1161/01.hyp.0000032940.33466.12

51. Anyaegbu E I, Dharnidharka V R. Hypertension in the teenager. Pediatr Clin NAm. (2014) 61:131-51. doi: 10.1016/j.pcl.2013.09.011

52. Palatini P. Sympathetic overactivity in hypertension: a risk factor for cardiovascular disease. Curr Hypertens Rep. (2001) 3:S3-9. doi: 10.1007/s11906-001-0065-Z

53. Frayn $\mathrm{K} \mathrm{N}$. Visceral fat and insulin resistance-causative or correlative? $\mathrm{Br} \mathrm{J}$ Nutr. (2000) 83 (Suppl. 1):S71-7. doi: 10.1017/s0007114500000982

54. Zhao Y, Wang L, Xue H, Wang H, Wang Y. Fast food consumption and its associations with obesity and hypertension among children: results from the baseline data of the childhood obesity study in china mega-cities. BMC Public Health. (2017) 17:933. doi: 10.1186/s12889-017-4952-x

Conflict of Interest: The authors declare that the research was conducted in the absence of any commercial or financial relationships that could be construed as a potential conflict of interest.

Copyright (c) 2021 Ye, Yi, Shao, Zhang, Zha, Yang, Xia, Ye and Song. This is an open-access article distributed under the terms of the Creative Commons Attribution License (CC BY). The use, distribution or reproduction in other forums is permitted, provided the original author(s) and the copyright owner(s) are credited and that the original publication in this journal is cited, in accordance with accepted academic practice. No use, distribution or reproduction is permitted which does not comply with these terms. 